\title{
(9)
}

\section{Prospective study on embolization of intracranial aneurysms with the pipeline device: the PREMIER study 1 year results}

\author{
Ricardo A Hanel, ${ }^{1}$ David F Kallmes, ${ }^{2}$ Demetrius Klee Lopes, ${ }^{3}$ Peter Kim Nelson, ${ }^{4}$ \\ Adnan Siddiqui, ${ }^{5}$ Pascal Jabbour, ${ }^{6}$ Vitor M Pereira, ${ }^{7}$ Istvan Szikora István, ${ }^{8}$ \\ Osama 0 Zaidat $\left(10,{ }^{9}\right.$ Chetan Bettegowda, ${ }^{10}$ Geoffrey P Colby $(1), 11$ \\ Maxim Mokin (1) ${ }^{12}$ Clemens Schirmer, ${ }^{13}$ Frank R Hellinger, ${ }^{14}$ Curtis Given II, \\ Timo Krings, ${ }^{16}$ Philipp Taussky (1) , ${ }^{17}$ Gabor Toth 이 , ${ }^{18}$ Justin F Fraser (1) , ${ }^{19}$ \\ Michael Chen, ${ }^{20}$ Ryan Priest, ${ }^{21}$ Peter Kan, ${ }^{22}$ David Fiorella $\left({ }^{20},{ }^{23}\right.$ Don Frei, ${ }^{24}$ \\ Beverly Aagaard-Kienitz, ${ }^{25}$ Orlando Diaz, ${ }^{26}$ Adel M Malek, ${ }^{27} \mathrm{C}$ Michael Cawley, ${ }^{28}$ \\ Ajit S Puri2
}

\begin{abstract}
- Additional material is published online only. To view please visit the journal online (http://dx.doi.org/10.1136/ neurintsurg-2019-015091).
\end{abstract}

For numbered affiliations see end of article.

\section{Correspondence to} Dr. Ricardo A Hanel, Baptist Neurological Institute, Lyerly Neurosurgery, Jacksonville, FL 32207, USA; rhanel@ lyerlyneuro.com

Received 6 May 2019 Revised 19 June 2019 Accepted 21 June 2019 Published Online First 15 July 2019

\section{Check for updates}

(C) Author(s) (or their employer(s)) 2020. Re-use permitted under CC BY-NC. No commercial re-use. See rights and permissions. Published by BMJ.

To cite: Hanel RA

Kallmes DF, Lopes DK, et al.

$\checkmark$ Neurolntervent Surg

2020;12:62-66.

\begin{abstract}
Background Preliminary clinical studies on the safety and efficacy of the pipeline embolization device (PED) for the treatment of small/medium aneurysms have demonstrated high occlusion rates with low complications.
\end{abstract}

Objective To evaluate the safety and effectiveness of the PED for treatment of wide necked small and medium intracranial aneurysms.

Methods PREMIER is a prospective, multicenter, single arm trial. Patients were treated with the PED for unruptured wide necked aneurysms, measuring $\leq 12 \mathrm{~mm}$ along the internal carotid artery or vertebral artery, between July 2014 and November 2015. At 1 year post-procedure, the primary effectiveness endpoint was complete occlusion (Raymond grade 1) without major parent vessel stenosis $(\leq 50 \%)$ or retreatment, and the primary safety endpoint was major stroke in the territory supplied by the treated artery or neurologic death.

Results A total of 141 patients were treated with PEDs (mean age 54.6 \pm 11.3 years, 87.9\% (124/141) women). Mean aneurysm size was $5.0 \pm 1.92 \mathrm{~mm}$, and $84.4 \%(119 / 141)$ measured $<7 \mathrm{~mm}$. PED placement was successful in $99.3 \%$ (140/141) of patients. Mean number of PEDs implanted per patient was 1.1 \pm 0.26 ; a single PED was used in $92.9 \%(131 / 141)$ of patients. At 1 year, 97.9\% (138/141) of patients underwent follow-up angiography with $76.8 \%(106 / 138)$ of patients having met the study's primary effectiveness endpoint. The combined major morbidity and mortality rate was $2.1 \%$ (3/140).

Conclusions Treatment of wide necked small/medium aneurysms with the PED results in high rates of complete occlusion without significant parent vessel stenosis and low rates of permanent neurologic complications. Trial registration NCT02186561.

\section{INTRODUCTION}

Although surgical clipping, coiling, and stent assisted coiling are well established treatment options for small and medium wide necked aneurysms, these modalities are limited by associated morbidity and/ or aneurysm recurrence rates. ${ }^{12}$ The advent of flow diverters (FD), such as the pipeline embolization device (PED) (Medtronic, Irvine, California, USA) has changed the landscape for intracranial aneurysm treatment by introducing a minimally invasive therapy that could be used to treat wide necked and large/giant aneurysms effectively. However, large and giant aneurysms only encompass a small fraction of all intracranial aneurysms (IAs), as approximately $80 \%$ of all unruptured IAs are small or medium in size $(\leq 12 \mathrm{~mm}) .^{3}$ Furthermore, the majority of ruptured IAs are smaller than $10 \mathrm{~mm}^{34}$

Several studies have examined the efficacy of the PED for small/medium IAs and shown preliminary promise of high occlusion rates with low morbidity and mortality. ${ }^{125-14}$ The IntrePED study reported outcomes for 793 patients (53\% of whom had small/medium aneurysms) and demonstrated an excellent safety profile. ${ }^{14}$ However, no prospective trial has yet tested the efficacy of PEDs specifically for small/medium wide necked aneurysms. The purpose of the Prospective Study on Embolization of Intracranial Aneurysms with the Pipeline Device (PREMIER) was to evaluate the safety and effectiveness of the PED in the treatment of wide necked intracranial aneurysms, measuring $\leq 12 \mathrm{~mm}$, located along the internal carotid artery (ICA) (up to the terminus) or the vertebral artery (VA) segment up to and including the posterior inferior cerebellar artery.

\section{METHODS}

Study design, enrollment and patient selection PREMIER (Clinical Trial Registry No NCT02186561) was a prospective, multicenter, single arm, interventional study. Between July 2014 and November 2015, 197 patients from 22 US participating sites and 1 Canadian center consented to achieve a total of 141 patients treated with PEDs. The study sample size was driven by the primary 
safety endpoint, defined as neurological death or major stroke in the territory supplied by the treated artery through 1 year; based on a performance goal of $15 \%$ and a postulated primary safety event rate of no more than 9\%, a sample size of 141 was required.

The major criterion for inclusion in the study was the presence of wide necked, unruptured IA arising from the ICA (all segments up to the carotid terminus) or VA (up to and including the posterior inferior cerebellar artery), measuring $\leq 12 \mathrm{~mm}$ in diameter, with neck $\geq 4 \mathrm{~mm}$ or a dome to neck ratio $\leq 1.5$. The major cause of patient exclusion was failure to meet preprocedure P2Y12 reaction units (PRU) (35/56). Patient inclusion and exclusion criteria are detailed in the online supplementary table I .

Aneurysm dimensions were measured using catheter based angiography and three-dimensional rotational angiogram images acquired at the time of patient screening. All images were submitted to an imaging screening committee and patients had to be considered appropriate for inclusion by at least two screening committee members prior to enrollment.

\section{Baseline assessments}

Prior to placement of the PED, patients underwent a baseline neurologic assessment using the modified Rankin Scale (mRS) and National InstitutesHealth Stroke Scale (NIHSS). Medical history, pre-existing conditions, and reason for treatment were documented. Baseline imaging consisted of CT angiography, MR angiography, or DSA, taken within 180 days prior to the procedure.

\section{Dual antiplatelet therapy}

Patients were tested for antiplatelet drug response using VerifyNow (Accumetrics, San Diego, California, USA) before PED implantation. PRU were required to be between 60 and 200 . Patients with a PRU value outside this range were excluded. Patients were placed on aspirin (minimum $81 \mathrm{mg} /$ day for 7 days) and clopidogrel (minimum $75 \mathrm{mg}$ /day for 7 days) prior to PED placement. Clopidogrel loading dose or clopidogrel substitution for prasugrel or ticagrelor was not allowed for study participants. After the procedure, patients were placed on aspirin (81-325 mg/day) for at least 6 months and $75 \mathrm{mg} /$ day of clopidogrel for at least 3 months.

\section{Study device and procedure}

The features of PED Classic and PED Flex have been previously described. ${ }^{15} 16$ PED Flex was introduced during the second half of the study, with no change in implant procedures. Procedures were performed under either general anesthesia or local anesthesia with sedation by using standard transfemoral or radial approaches. Intravenous heparin was administered at 50-100 U/ $\mathrm{kg}$ to achieve an activated clotting time $>200 \mathrm{~s}$. A 0.027 inch microcatheter (Marksman Catheter; Medtronic, Irvine, California) was used, with additional PEDs allowed, as needed, to completely cover the neck of the aneurysm. Additional coils were used at the operators' discretion. For utilization of PED Flex, the operators were required to have used the device in at least three previous cases.

\section{Follow-up assessments}

Patients underwent neurological assessment at 30 days, 6 months, and 1 year post-procedure. Follow-up DSA was mandatory at 1 year. All images were submitted for assessment by an independent core laboratory. Images were evaluated for degree of aneurysm occlusion according to the Roy-Raymond scale, ${ }^{17}$ the presence and degree of parent vessel stenosis according to the methods of Samuels et al, ${ }^{18}$ and the occurrence of implant migration.

\section{Safety reporting}

Investigators were requested to report all negative changes in health, and to judge the relationship of the adverse event to both the PED and the placement procedure. All serious adverse events were reviewed and adjudicated by an independent clinical events committee.

\section{Study endpoints}

The primary effectiveness endpoint was complete occlusion (Raymond Scale I) of the target IA without significant $(\leq 50 \%)$ stenosis of the parent artery or retreatment through 1 year follow-up. The primary safety endpoint was incidence of major stroke (ischemic or hemorrhagic) in the territory supplied by the treated artery, defined as an increase in NIHSS score by 4 points or neurologic death within 1 year after treatment. Secondary endpoints included hemorrhagic complications at 30 days and device deployment success rate. Additional data collected at 1 year included device related neurologic adverse events, mRS score, recurrence and retreatment rates, procedural time, and number of PEDs utilized.

\section{Statistical analysis}

Demographic, baseline, and procedural characteristics were summarized and reported as mean $\pm S D$, median, and minimum and maximum values for continuous variables. Categorical data were summarized using numbers and percentages. In addition to reporting outcomes based on available data for missing data at the 1 year follow-up, a multiple imputation analysis was performed, which included the primary effectiveness endpoint, primary safety endpoint, and the two secondary safety endpoints. The variables selected for the multiple imputation analysis were age, gender, aneurysm maximal diameter, and parent artery location. Statistical analysis was performed using R (V.3.0 and above, R Foundation for Statistical Computing, Vienna, Austria) and SAS (V.9.0 and above, SAS Institute, Cary, North Carolina, USA).

\section{RESULTS}

\section{Patient and aneurysm characteristics}

A total of 141 patients were treated; 124 (87.9\%) were women. Mean age was 54.6 \pm 11.3 years. Median target aneurysm size was $4.6 \mathrm{~mm}$ (mean $5.0 \pm 1.92 \mathrm{~mm}$ ) with median neck size of $3.7 \mathrm{~mm}$ (mean $4.0 \pm 1.42 \mathrm{~mm}$ ). Among the 141 aneurysms, 119 $(84.4 \%)$ measured $<7 \mathrm{~mm}$ in size and $22(15.6 \%)$ measured $7-12 \mathrm{~mm}$ on the largest diameter.

There were 136 (96.5\%) aneurysms with saccular morphology, of which $47(34.6 \%)$ had side branch involvement. The remaining $5(3.5 \%)$ aneurysms were fusiform. A total of 134 (95.0\%) aneurysms were located along the ICA, with the remaining 7 (5.0\%) at the V4 segment of the VA. Most aneurysms in the ICA were located at the C6 ophthalmic segment $(74.6 \%, 100 / 134)$ and C7 communicating segment (14.2\%, 19/134). Patient baseline characteristics, medical history, and aneurysm characteristics are summarized in table 1.

Reasons for treatment of aneurysms are summarized in the online supplementary table II. The most common reason for treatment was patient preference $(63.1 \%, 89 / 141)$. A summary of aneurysm risk factors is reported in the online supplementary 
Table 1 Baseline patient and aneurysm characteristics

\begin{tabular}{|c|c|}
\hline \multicolumn{2}{|l|}{ Characteristic } \\
\hline \multicolumn{2}{|l|}{ Demographics $(n=141)$} \\
\hline Age (years) (mean $\pm S D$ ) & $54.6 \pm 11.3$ \\
\hline Women (n (\%)) & $124(87.9)$ \\
\hline Baseline mRS (mean \pm SD (range)) & $0.2 \pm 0.5(0-2)$ \\
\hline Baseline NIHSS (mean \pm SD (range)) & $0.1 \pm 0.6(0-4)$ \\
\hline \multicolumn{2}{|l|}{ Medical history ( $n=141)(n(\%))$} \\
\hline Hypertension & $72(51.1)$ \\
\hline Hyperlipidemia & $54(38.3)$ \\
\hline Diabetes mellitus & $16(11.3)$ \\
\hline Obesity & $24(17.0)$ \\
\hline Cerebral atherosclerosis & $1(0.7)$ \\
\hline Atrial fibrillation & $7(5.0)$ \\
\hline Myocardial infarction & $2(1.4)$ \\
\hline Coronary artery disease & $9(6.4)$ \\
\hline Sleep apnea & $16(11.3)$ \\
\hline Alcohol use & $3(2.1)$ \\
\hline \multicolumn{2}{|l|}{ Smoking history (n (\%)) } \\
\hline Never smoked, or has not smoked within 10 years & $79(56.0)$ \\
\hline Not a current smoker, but has smoked within 10 years & $21(14.9)$ \\
\hline Current smoker, $<1$ pack/day & $27(19.1)$ \\
\hline Current smoker, $\geq 1$ pack/day & $14(9.9)$ \\
\hline \multicolumn{2}{|l|}{ Aneurysms characteristics (median $($ mean $\pm S D)$ ) } \\
\hline Aneurysm size (mm) & $4.6(5.0 \pm 1.9)$ \\
\hline Neck width (mm) & $3.7(4.0 \pm 1.4)$ \\
\hline Dome to neck ratio & $1.1(1.1 \pm 0.3)$ \\
\hline \multicolumn{2}{|l|}{ Aneurysm location (n/N (\%)) } \\
\hline ICA & 134/141 (95.0) \\
\hline ICA-petrous segment (C2) & $1 / 134(0.7)$ \\
\hline ICA-clinoid segment (C4) & $11 / 134(8.2)$ \\
\hline ICA-cavernous segment (C5) & $3 / 134(2.2)$ \\
\hline ICA-ophthalmic segment (C6) & 100/134 (74.6) \\
\hline ICA-PCom segment (C7) & 19/134 (14.2) \\
\hline Vertebral artery & $7 / 141(5)$ \\
\hline
\end{tabular}

ICA, internal carotid artery; mRS, modified Rankin Scale; NIHSS, National Institute of Health Stroke Scale; PCom, posterior communicating artery.

table III. Aneurysm multiplicity was the most prevalent aneurysm risk factor $(37.6 \%, 53 / 141)$.

\section{Procedure}

Successful device deployment was reported in 140/141 cases (99.3\%). In one patient, a PED was not successfully implanted during the first procedure attempt and was successfully placed during a second procedure. A single PED was used in 131 (92.9\%) patients, and multiple PEDs were used for complete neck coverage in 9 (6.4\%) patients. The mean number of devices used per patient was $1.1 \pm 0.3$ (range $0-2$ ). Mean total procedural time was $78.4 \pm 40.3 \mathrm{~min}$ (range 20-217), and mean time from first PED introduction to last PED delivery system removal was $14.3 \pm 15.1 \mathrm{~min}$. PED Classic was used in $64(45.7 \%)$ patients while PED Flex was used in $77(55.0 \%)$ cases. In 5 (3.5\%) cases, the operators chose adjuvant use of coils. Core laboratory adjudication demonstrated complete aneurysm neck coverage by

\begin{tabular}{|c|c|}
\hline Characteristic & $n=141$ \\
\hline Successful device deployment to target site (n (\%)) & $140(99.3)$ \\
\hline Procedure time $(\min )(\operatorname{mean} \pm \mathrm{SD}($ range)$)$ & $78.4 \pm 40.3(20-217)$ \\
\hline $\begin{array}{l}\text { First pipeline introduction to last device removal time } \\
(\min )(\text { mean } \pm S D \text { (range)) }\end{array}$ & $14.3 \pm 15.1(1-92)$ \\
\hline No of pipeline devices per patient (mean \pm SD (range)) & $1.1 \pm 0.3(1-2)$ \\
\hline Patients with multiple pipeline devices ( $\mathrm{n}(\%))$ & $9(6.4)$ \\
\hline \multicolumn{2}{|l|}{ Pipeline devices used* (n (\%)) } \\
\hline Pipeline embolization device Classic & $64 / 140(45.7)$ \\
\hline Pipeline embolization device Flex & $77 / 140(55.0)$ \\
\hline Balloon used (n (\%)) & $31(22.0)$ \\
\hline \multicolumn{2}{|l|}{ Adjunctive devices used (per imaging core lab) (n (\%)) } \\
\hline Coils & $5(3.5)$ \\
\hline None & $136(96.5)$ \\
\hline Complete wall apposition (n (\%)) & $119(84.4)$ \\
\hline Entire neck covered (n (\%)) & $139(98.6)$ \\
\hline
\end{tabular}

${ }^{*}$ One patient had both type of devices implanted.

PED in 139 (98.6\%) cases with complete wall apposition in 119 (84.4\%). Procedural details are described in table 2.

\section{Primary effectiveness endpoint}

Of the total 141 patients, 138 (97.9\%) underwent 1 year follow-up angiography with $81.9 \%(113 / 138)$ of the aneurysms demonstrating complete occlusion (Raymond-Roy grade 1). In the intent to treat population, the primary effectiveness endpoint was met in $76.8 \%(106 / 138)$ of patients. Thirty-two patients $(23.2 \%, 32 / 138)$ failed to reach the primary effectiveness endpoint. Reasons for primary effectiveness endpoint failure are described in table 3 . There were three patients with missing follow-up images: one died, one refused follow-up, and one agreed to have clinical follow-up but not imaging follow-up.

\section{Primary safety endpoint}

Of 140 patients with available data, $3(2.1 \%)$ experienced a primary safety endpoint event (major stroke), with 1 leading to neurological death. These results are summarized in table 4. In one patient, symptoms of a left hemispheric event occurred 15 days after treatment. Head CT revealed an intraparenchymal hemorrhage (unrelated to aneurysm rupture). A second patient presented with seizure and left-sided weakness 165 days after the procedure. Head CT revealed an infarct involving the right middle cerebral artery territory. The patient had discontinued dual antiplatelets a few weeks before this event. A third patient developed a right hemispheric syndrome a few hours after treatment. Head CT demonstrated a large intraparenchymal

Table 3 Summary of failure reasons for primary effectiveness endpoint

\begin{tabular}{lc}
\hline Reasons for primary effectiveness endpoint failure & $\mathrm{n}=138$ \\
\hline Residual aneurysm $(\mathrm{n}(\%))$ & $22(15.9)$ \\
\hline Residual neck $(\mathrm{n}(\%))$ & $3(2.2)$ \\
\hline Stenosis $>50 \%(\mathrm{n}(\%))$ & $4(2.9)$ \\
\hline Target aneurysm retreatment $(\mathrm{n}(\%))$ & $4(2.9)$ \\
\hline Total $(\mathrm{n}(\%))$ & $32(23.2)^{*}$ \\
\hline${ }^{*}$ One subject had both incomplete occlusion and retreatment.
\end{tabular}


Table 4 Primary outcomes and secondary safety outcomes at 1 year post-procedure

\begin{tabular}{lcc}
\hline Event & $\begin{array}{l}\text { Observed data (n/N } \\
(\%))\end{array}$ & $\begin{array}{c}\text { Multiple } \\
\text { imputation (n=141)* (\%) }\end{array}$ \\
\hline Primary effectiveness outcome & $106 / 138(76.8)$ & 76.7 \\
\hline Complete aneurysm occlusion without significant parent artery stenosis $(\leq 50 \%)$ or retreatment & $3 / 140(2.1)$ & 2.2 \\
\hline $\begin{array}{l}\text { Primary safety outcome } \\
\text { Major stroke in the territory supplied by the treated artery or neurological death at 1 year post-procedure }\end{array}$ & 0.0 \\
\hline $\begin{array}{l}\text { Secondary outcomes } \\
\text { Major stroke in the territory supplied by the treated artery or neurological death due to procedural complications at }\end{array}$ & $0 / 140(0.0)$ \\
\hline $\begin{array}{l}\text { 30 days post-procedure } \\
\text { Delayed intracerebral hemorrhage }>30 \text { days post-procedure }\end{array}$ & $1 / 140(0.7)$ \\
\hline
\end{tabular}

*Multiple imputation for patients missing endpoint evaluation.

hemorrhage with significant mass effect, and midline shift with subfalcine and uncal herniation, leading to the patient's death post-procedure.

\section{Other outcomes}

There were no cases of intraoperative aneurysm rupture, delayed aneurysm rupture, or documented recurrence after complete aneurysm occlusion. At the 1 year follow-up, $137 / 139$ (98.6\%) patients had an mRS score of 0-2, and 4/141 (2.8\%) underwent retreatment. The secondary safety endpoint of delayed intracerebral hemorrhage 31 days to 1 year post-procedure occurred in 1 patient $(0.7 \%)$. The clinical events committee adjudicated this event as non-serious, and device related.

\section{DISCUSSION}

PREMIER is the first prospective multicenter study to evaluate the use of FDs in small/medium, unruptured IAs located in the ICA and VA. Treatment of these aneurysms with PEDs results in high rates of complete occlusion with low morbidity, mortality, recurrence, and retreatment, suggesting that the PED is a safe and effective alternative to microsurgery and conventional endovascular techniques in such cases.

While small/medium aneurysms are most often treated with coils, wide necked aneurysms are difficult to coil due to the increased risk of coil migration or protrusion into the parent vessel. $^{19} 20$ The PED shape optimizes its ability to cover the aneurysm neck and results in high occlusion rates without the risks associated with microsurgery, and with higher rates of complete occlusion than other endovascular techniques, such as coil embolization with or without stent or balloon assistance, in wide necked aneurysms. ${ }^{21}$ Moreover, the PED is often used as a standalone therapy, which simplifies the procedure by not requiring aneurysmal catherization, ${ }^{9}$ and reduces procedure time. ${ }^{22}$ As the PED is deployed without the need to access the aneurysmal sac, the risk of intraoperative rupture may be substantially reduced. ${ }^{7-9}$ The PREMIER study supports this finding, as no intraoperative ruptures were reported. This procedural advantage may be especially important for the treatment of small/medium aneurysms, as the risk of intraoperative rupture during coiling has been reported to be higher compared with large aneurysms. ${ }^{2324}$

The Pipeline for Uncoilable or Failed Aneurysms (PUFS) study previously demonstrated the safety and efficacy of the PED for aneurysms $>10 \mathrm{~mm}$ located in the ICA, proximal to the posterior communicating segment, with an occlusion rate of $95 \%$ at 5 years and no episodes of recurrence after complete occlusion. $^{2526}$ However, approximately $80 \%$ of all aneurysms were small or medium in size $(\leq 12 \mathrm{~mm}),{ }^{3}$ and the majority of ruptured aneurysms were $<10 \mathrm{~mm} .{ }^{34}$ A handful of studies have begun exploring the use of the PED for the treatment of small and medium wide necked aneurysms and have shown a promising safety and effectiveness profile, ${ }^{12568911-132728}$ although these studies are limited by their retrospective design, single center series, and lack of external adjudication of adverse events or angiography. The incidence of thromboembolic events was reported as $7.3-8.7 \%$ of patients. ${ }^{28}$ Mortality rates ranged from $0 \%$ to $2.3 \%,{ }^{12} 282728$ and occlusion rates ranged from $70.0 \%$ to $91.7 \% .^{125682728}$ One major international retrospective study, IntrePED, analyzed the results of 793 patients with 906 aneurysms treated with PEDs in 17 centers. ${ }^{15}$ There were $473(52.2 \%)$ small aneurysms ( $<10 \mathrm{~mm}), 349$ (38.5\%) of which were located in the ICA. The combined neurologic morbidity and mortality rate for unruptured aneurysms $<10 \mathrm{~mm}$ in IntrePED was $4.1 \%$ compared with the higher rate of $9.2 \%$ seen with larger aneurysms.

In terms of the metal coverage required for effective aneurysm treatment, the PREMIER study had a mean device utilization of 1.1 , in contrast with three devices used in PUFS. ${ }^{25}$ Multiple devices were used in only 6.4\% of cases in PREMIER. Occlusion rates at 1 year for PUFS (87\%) were slightly higher than PREMIER, but are difficult to compare statistically due to differences in inclusion criteria and aneurysm characteristics. In a recent meta-analysis of the FD literature ${ }^{29}$ the rate of complete occlusion in 1645 aneurysms was lower than the PREMIER study at $76 \%$ (95\% CI $70 \%$ to $81 \%)$.

PREMIER is the first FD study to exclude patients from enrollment based on pretreatment PRU levels outside a prespecified range at all treating sites. Our study reported lower ischemic complication rates, but similar hemorrhagic complications compared with IntrePED. This could be partially explained by collective and individual learning curves with PEDs and introduction of the PED Flex system. However, limiting the use of FDs to patients responsive to antiplatelet therapy seems to yield better results. It remains unknown whether actively monitoring platelet function and adjusting antiplatelet therapy similarly leads to improved outcomes. Although this requires further studies, device surface modification has been proposed as a possible strategy to reduce thromboembolic complications, such as PED Shield, which includes a surface phosphorylcholine biocompatible polymer that has the potential to reduce thromboembolic complications. $^{3031}$

A major limitation of this study is the single am nature and lack of a comparison group with which to compare outcomes. Furthermore, almost $75 \%$ of the aneurysms treated were located in the ophthalmic segment of the ICA; further location focused studies may be needed to demonstrate the effectiveness of PED 
throughout the cerebral vasculature. Lastly, PREMIER examines a specific subset of difficult to coil aneurysms, but the lack of large, giant, or unruptured aneurysms means that its results should only be generalized to small/medium wide necked aneurysms.

\section{CONCLUSION}

PREMIER is the first prospective, independently adjudicated imaging and clinical outcomes, multicenter study assessing the safety and efficacy of PEDs for the treatment of unruptured IAs measuring $\leq 12 \mathrm{~mm}$ located along the ICA and VA. The present findings provide evidence of high procedural success and high complete occlusion, with low morbidity, mortality, recurrence, and retreatment. Our findings suggest that, when a decision is made to treat these lesions, the PED is a safe and effective alternative to microsurgery and conventional endovascular techniques.

\section{Author affiliations}

${ }^{1}$ Baptist Neurological Institute, Lyerly Neurosurgery, Jacksonville, Florida, USA 'Departments of Neurosurgery and Radiology, Mayo Clinic, Rochester, Minnesota, USA

${ }^{3}$ Brain and Spine Institute, Advocate Aurora Health, Chicago, Illinois, USA

${ }^{4}$ Interventional Radiology, New York University Langone Medical Center, New York, New York, USA

${ }^{5}$ Departments of Neurosurgery and Radiology, University at Buffalo Jacobs School of Medicine and Biomedical Sciences, Buffalo, New York, USA

${ }^{6}$ Department of Neurosurgery, Thomas Jefferson University Hospital, Philadelphia, Pennsylvania, USA

${ }^{7}$ Departments of Medical Imaging and Surgery, Division of Neuroradiology and Neurosurgery, Toronto Western Hospital, Toronto, Ontario, Canada

${ }^{8}$ Department of Neuroradiology, National Institute of Neurosciences, Budapest, Hungary

${ }^{9}$ Neuroscience Institute, St Vincent Mercy Hospital, Toledo, Ohio, USA

${ }^{10}$ Department of Neurosurgery, Johns Hopkins University School of Medicine, Baltimore, Maryland, USA

${ }^{11}$ Department of Neurosurgery, University of California Los Angeles, Los Angeles, California, USA

${ }^{12}$ Department of Neurosurgery, University of South Florida, Tampa, Florida, USA

${ }^{13}$ Department of Neurological Surgery, Geisinger Clinic, Danville, Pennsylvania, USA

${ }^{14}$ Department of Radiology, Florida Hospital Neuroscience Institute, Winter Park,

Florida, USA

${ }^{15}$ Department of Radiology, Baptist Health Lexington, Lexington, Kentucky, USA

${ }^{16}$ Department of Medical Imaging, Toronto Western Hospital, Toronto, Ontario, Canada

${ }^{17}$ Department of Neurosurgery, University of Utah, Salt Lake City, Utah, USA

${ }^{18}$ Cerebrovascular Center, Cleveland Clinic, Cleveland, Ohio, USA

${ }^{19}$ Department of Neurological Surgery, University of Kentucky, Lexington, Kentucky, USA

${ }^{20}$ Department of Neurological Surgery, Rush University Medical Center, Chicago, Illinois, USA

${ }^{21}$ Charles T Dotter Department of Interventional Radiology, Dotter Interventional Institute, Oregon Health and Science University, Portland, Oregon, USA

${ }^{22}$ Department of Neurosurgery, Baylor College of Medicine, Houston, Texas, USA

${ }^{23}$ Department of Neurosurgery, Cerebrovascular Center, Stony Brook University, Stony Brook, New York, USA

${ }^{24}$ Department of Neuroradiology, Swedish Medical Center, Denver, Colorado, USA

${ }^{25}$ Department of Neurological Surgery, University of Wisconsin Madison School of

Medicine and Public Health, Madison, Wisconsin, USA

${ }^{26}$ Division of Interventional Neuroradiology, Houston Methodist Research Institute, Houston, Texas, USA

${ }^{27}$ Department of Neurosurgery, Tufts Medical Center, Tufts University School of Medicine, Boston, Massachusetts, USA

${ }^{28}$ Departments of Neurointerventional Radiology and Neurosurgery, Emory University School of Medicine, Atlanta, Georgia, USA

${ }^{29}$ New England Center for Stroke Research, Department of Radiology, University of Massachusetts Medical School, Worcester, Massachusetts, USA

Correction notice Since this paper was first published online, the author Sam Zaidat should be listed as Osama 0 Zaidat.

Acknowledgements The authors acknowledge Medtronic for providing editing support for this manuscript.

Contributors All authors were involved and made substantial contributions to the conception or design of the work, or the acquisition, analysis, or interpretation of the data; drafting the work or revising it critically for important intellectual content; final approval of the version published; and agreement to be accountable for all aspects of the work in ensuring that questions related to the accuracy or integrity of any part of the work are appropriately investigated and resolved.

Funding The PREMIER study was supported by Medtronic, Inc.

Competing interests RH serves as a consultant for Medtronic, Stryker, Codman, and Microvention and is a stock holder of In Neuroco. DFK is president of Marblehead Medical and has patent pending in balloon catheter technologies. He has received research support from Medtronic, MicroVention, NeuroSave, Neurogami, Sequent Medical, NeuroSigma, and Insera, and also serves on the Scientific Advisory Board for Triticum and Boston Scientific. PKN is a consultant for Medtronic. AS is a modest consultant for Amnis Therapeutics, Boston Scientific, Canon Medical Systems USA, Cerebrotech Medical Systems, Claret Medical, Corindus, Endostream Medical, Guidepoint Global Consulting, Imperative Care, Integra, Rapid Medical, Rebound Therapeutics Corp, Silk Road Medical, StimMed, Stryker, Three Rivers Medical, VasSol, and WL Gore and Associates. He is also a consultant and serves on the national PI/Steering Committee for Cerenovus, Medtronic, MicroVention, and Penumbra. He serves on the National PI/Steering Committee for the POSITIVE Trial for the Medical University of South Carolina and as DSMB Chair for the HEAT Trial for Northwest University and has ownership interest in Amnis Therapeutics, Apama Medical, BlinkTBI, Buffalo Technology Partners, Cardinal Health, Cerebrotech Medical Systems, Claret Medical, Cognition Medical, Endostream Medical Ltd, Imperative Care, International Medical Distribution Partners, Rebound Therapeutics Corp, Silk Road Medical, StimMed, Synchron, Three Rivers Medical, and Viseon. PJ serves as a consultant for Medtronic. VMP serves as a consultant/Steering Committee member for Stryker, Penumbra, and Balt, and as a consultant for Medtronic and Neurovasc, and receives a research grant from Philips. SZ serves as a scientific consultant regarding trial design and conduct to Medtronic. GPC serves as a consultant for Medtronic, Microvention-Terumo, and Stryker. MM serves as a consultant for Cerebrotech, Imperative Care, and Penumbra. CS has received honoraria from the American Association of Neurological Surgeons and Toshiba, and has ownership interest in NTI. FRH serves on the Speakers' Bureau for Medtronic and as a consultant for Penumbra and Cordis Neurovascular (Johnson \& Johnson). CG serves as a consultant, proctor, and on the Speakers' Bureau for Medtronic and Stryker, and is on the Speaker's Bureau for Genentech. PT serves as a consultant for Covidien. GT serves as a consultant for Dynamed EBSCO. JFF is an equity interest holder for Fawkes Biotechnology, LLC, and is a consultant for Stream Biomedical and for Medtronic. MC is a consultant for Medtronic, Stryker, Penumbra, Genentech, and GE. PK is a consultant for Stryker Neurovascular, Medtronic, and Cerenovus. DFi is a consultant and has received research support from Microvention/Terumo. DFr is a consultant and on the Speakers' Bureau for Penumbra, Stryker Neurovascular, Genentech, MicroVention, and Codman. OD serves as a proctor for Microvention/ Terumo. AMM is a cofounder, investor, and shareholder of CereVasc. ASP consults for and has received research grants from Medtronic Neurovascular and Stryker Neurovascular.

\section{Patient consent for publication Not required.}

Ethics approval The study was performed in compliance with the WorldMedical Association's Declaration of Helsinki. Each institutional review boardapproved the protocol and the informed consent form.

Provenance and peer review Not commissioned; externally peer reviewed.

Open access This is an open access article distributed in accordance with the Creative Commons Attribution Non Commercial (CC BY-NC 4.0) license, which permits others to distribute, remix, adapt, build upon this work non-commercially, and license their derivative works on different terms, provided the original work is properly cited, appropriate credit is given, any changes made indicated, and the use is non-commercial. See: http://creativecommons.org/licenses/by-nc/4.0/.

\section{ORCID iDs}

Osama O Zaidat http://orcid.org/0000-0003-4881-4698

Geoffrey P Colby http://orcid.org/0000-0002-3376-0933

Maxim Mokin http://orcid.org/0000-0003-4270-8667

Philipp Taussky http://orcid.org/0000-0001-9247-0146

Gabor Toth http://orcid.org/0000-0002-3646-3635

Justin F Fraser http://orcid.org/0000-0002-5980-3989

David Fiorella http://orcid.org/0000-0002-2677-8780

\section{REFERENCES}

1 Chalouhi N, Zanaty M, Whiting A, et al. Safety and efficacy of the Pipeline Embolization Device in 100 small intracranial aneurysms. J Neurosurg 2015;122:1498-502

2 Griessenauer CJ, Ogilvy CS, Foreman PM, et al. Pipeline Embolization Device for small paraophthalmic artery aneurysms with an emphasis on the anatomical relationship of ophthalmic artery origin and aneurysm. I Neurosurg 2016;125:1352-9 
3 Wiebers DO, Whisnant JP, Huston J, et al. Unruptured intracranial aneurysms: natural history, clinical outcome, and risks of surgical and endovascular treatment. Lancet 2003;362:103-10

4 International Study of Unruptured Intracranial Aneurysms Investigators. Unruptured intracranial aneurysms--risk of rupture and risks of surgical intervention. N Engl J Med 1998;339:1725-33.

5 Chalouhi N, Daou B, Barros G, et al. Matched comparison of flow diversion and coiling in small, noncomplex intracranial aneurysms. Neurosurgery 2017:81:92-97.

6 Deutschmann HA, Wehrschuetz M, Augustin M, et al. Long-term follow-up after treatment of intracranial aneurysms with the Pipeline embolization device: results from a single center. AJNR Am J Neuroradiol 2012;33:481-6.

7 Griessenauer CJ, Ogilvy CS, Foreman PM, et al. Pipeline Embolization Device for small intracranial aneurysms: evaluation of safety and efficacy in a multicenter cohort. Neurosurgery 2017;80:579-87.

8 Lin LM, Colby GP, Kim JE, et al. Immediate and follow-up results for 44 consecutive cases of small $(<10 \mathrm{~mm})$ internal carotid artery aneurysms treated with the pipeline embolization device. Surg Neurol Int 2013;4:114.

9 Lylyk P, Miranda C, Ceratto R, et al. Curative endovascular reconstruction of cerebral aneurysms with the pipeline embolization device: the Buenos Aires experience. Neurosurgery 2009;64:632-43.

10 Chalouhi N, Zanaty M, Whiting A, et al. Treatment of ruptured intracranial aneurysms with the pipeline embolization device. Neurosurgery 2015;76:165-72. discussion 72.

11 Dabus G, Grossberg JA, Cawley CM, et al. Treatment of complex anterior cerebral artery aneurysms with Pipeline flow diversion: mid-term results. J Neurointerv Surg 2017;9:147-51.

12 Lin N, Brouillard AM, Keigher KM, et al. Utilization of Pipeline embolization device for treatment of ruptured intracranial aneurysms: US multicenter experience. $J$ Neurointerv Surg 2015;7:808-15.

13 Mazur MD, Kilburg C, Wang V, et al. Pipeline embolization device for the treatment of vertebral artery aneurysms: the fate of covered branch vessels. J Neurointerv Surg 2016;8:1041-7.

14 Kallmes DF, Hanel R, Lopes D, et al. International retrospective study of the pipeline embolization device: a multicenter aneurysm treatment study. AJNR Am J Neuroradiol 2015:36:108-15.

15 Aguilar-Salinas P, Brasiliense LB, Gonsales D, et al. Evaluation of Pipeline Flex delivery system for the treatment of unruptured aneurysms. Expert Rev Med Devices 2016;13:885-97.

16 Eller JL, Dumont TM, Sorkin GC, et al. The Pipeline embolization device for treatment of intracranial aneurysms. Expert Rev Med Devices 2014;11:137-50.
17 Roy D, Milot G, Raymond J. Endovascular treatment of unruptured aneurysms. Stroke 2001;32:1998-2004.

18 Samuels OB, Joseph GJ, Lynn MJ, et al. A standardized method for measuring intracranial arterial stenosis. AJNR Am J Neuroradiol 2000;21:643-6.

19 Hope JK, Byrne JV, Molyneux AJ. Factors influencing successful angiographic occlusion of aneurysms treated by coil embolization. AJNR Am J Neuroradiol 1999;20:391-9.

20 Pumar JM, Mosqueira A, Cuellar $\mathrm{H}$, et al. Expanding the use of flow diverters beyond their initial indication: treatment of small unruptured aneurysms. J Neurointerv Surg 2018; 10:245-8.

21 Nelson PK, Lylyk P, Szikora I, et al. The pipeline embolization device for the intracranial treatment of aneurysms trial. AJNR Am J Neuroradio/ 2011;32:34-40.

22 Chalouhi N, McMahon JF, Moukarzel LA, et al. Flow diversion versus traditional aneurysm embolization strategies: analysis of fluoroscopy and procedure times. $J$ Neurointerv Surg 2014;6:291-5.

23 Chowdhury T, Cappellani RB, Sandu N, et al. Perioperative variables contributing to the rupture of intracranial aneurysm: an update. ScientificWorldJournal 2013;2013:396404.

24 Mitchell PJ, Muthusamy S, Dowling R, et al. Does small aneurysm size predict intraoperative rupture during coiling in ruptured and unruptured aneurysms? I Stroke Cerebrovasc Dis 2013;22:1298-303.

25 Becske T, Kallmes DF, Saatci I, et al. Pipeline for uncoilable or failed aneurysms: results from a multicenter clinical trial. Radiology 2013;267:858-68.

26 Becske T, Brinjikji W, Potts MB, et al. Long-term clinical and angiographic outcomes following pipeline embolization device treatment of complex internal carotid artery aneurysms: five-year results of the pipeline for uncoilable or failed aneurysms trial. Neurosurgery 2017;80:40-8.

27 Chalouhi N, Starke RM, Yang S, et al. Extending the indications of flow diversion to small, unruptured, saccular aneurysms of the anterior circulation. Stroke 2014:45:54-8

28 Griessenauer CJ, Ogilvy CS, Foreman PM, et al. Pipeline Embolization Device for small intracranial aneurysms: evaluation of safety and efficacy in a multicenter cohort. Neurosurgery 2017;80:579-587.

29 Brinjikji W, Murad MH, Lanzino G, et al. Endovascular treatment of intracranial aneurysms with flow diverters: a meta-analysis. Stroke 2013;44:442-7.

30 Martínez-Galdámez M, Lamin SM, Lagios KG, et al. Treatment of intracranial aneurysms using the pipeline flex embolization device with shield technology: angiographic and safety outcomes at 1-year follow-up. J Neurointerv Surg 2019;11.

31 Pierot L, Spelle L, Berge J, et al. Feasibility, complications, morbidity, and mortality results at 6 months for aneurysm treatment with the Flow Re-Direction Endolumina Device: report of SAFE study. J Neurointerv Surg 2018;10:765-70. 